\title{
Reversible Visualization for Synchrotron Radiation Using Photochromic Dye and Photostimulable Phosphor Composite Film
}

\author{
Kenji Kinashi, ${ }^{1}$ Kazuya Jimbo, ${ }^{2}$ Takahiro Okabe, ${ }^{2}$ Sono Sasaki, ${ }^{3}$ and Hiroyasu Masunaga ${ }^{4}$ \\ ${ }^{1}$ Graduate School of Science and Technology, Department of Macromolecular Science and Engineering, Kyoto Institute of Technology, \\ Matsugasaki, Sakyo, Kyoto 606-8585, Japan \\ ${ }^{2}$ NISSHA Printing Co., Ltd., 3 Mibu, Hanai, Nakagyo, Kyoto 604-8551, Japan \\ ${ }^{3}$ Graduate School of Science and Technology, Department of Biobased Materials Science, Kyoto Institute of Technology, \\ Matsugasaki, Sakyo, Kyoto 606-8585, Japan \\ ${ }^{4}$ Japan Synchrotron Radiation Research Institute, 1-1-1 Kouto, Sayo, Hyogo 679-5198, Japan \\ Correspondence should be addressed to Kenji Kinashi; kinashi@kit.ac.jp
}

Received 17 July 2014; Accepted 16 October 2014; Published 11 November 2014

Academic Editor: Leonardo Palmisano

Copyright @ 2014 Kenji Kinashi et al. This is an open access article distributed under the Creative Commons Attribution License, which permits unrestricted use, distribution, and reproduction in any medium, provided the original work is properly cited.

The study reported herein is undertaken to visualize reversibly synchrotron radiation by using a composite film comprised of two components: a photochromic SP with the conversion characteristics of UV-to-visible color and PSP BaFCl:Eu ${ }^{2+}$ particles with the conversion characteristics of X-rays-to-UV emission.

\section{Introduction}

Almost immediately following their discovery by W. C. Röntgen in 1895, X-rays have been used for medical imaging. In present-day medical radiography, shadow images are made of the internal structures of the human body by placing the patient between an X-ray source and an X-ray detector. Currently, there are two different types of X-ray detectors for direct digital image acquisition: the chargecoupled device (CCD) and the X-ray storage phosphor plate. Most commercially available digital systems are CCD systems, and the image is displayed almost immediately on a computer monitor after exposure of the sensor. However, these systems have several disadvantages, such as a relatively bulky detector, the need for a wire connection between the sensor and the computer, a small active area of photon detection, and a relatively narrow dynamic range. On the other hand, the X-ray storage phosphor plate is an easy structure including its photostimulable phosphor (PSP) particles dispersed in a polymer binder and coated onto a plastic base. X-ray storage phosphors, which can absorb X-rays and convert the absorbed energy efficiently into ultraviolet (UV) or visible emission, are used in the application of light intensity measurement under continuous $\mathrm{X}$-ray radiation. A high-performance X-ray storage phosphor should be a high-density material or contain heavy elements because the absorption coefficient increases strongly with the atomic number [1]. Europium doped barium fluorohalides $\mathrm{BaFX}: \mathrm{Eu}^{2+}(\mathrm{X}=\mathrm{Br}, \mathrm{Cl})$ have a number of favorable properties that make them suitable for use as $\mathrm{X}$-ray storage phosphor in detection systems developed for two-dimensional X-ray imaging in the fields of radiography and crystallography [2, $3]$.

Photochromic spiropyran dyes are currently used in many commercial applications, such as ophthalmic lenses, sunglasses [4], optical memory, and photooptical switching devices [5], due to their reversible colored-decolored reaction under UV/visible light [6-8]. Spiropyran dyes consist of two heterocyclic moieties linked together by a sp3 spirocarbon atom. Irradiation of UV light and/or temperature increase causes cleavage of the relatively weak spirocarbon-oxygen (C-O) bond followed by conformational rearrangement, leading finally to ring-opened colored isomers, the so-called 


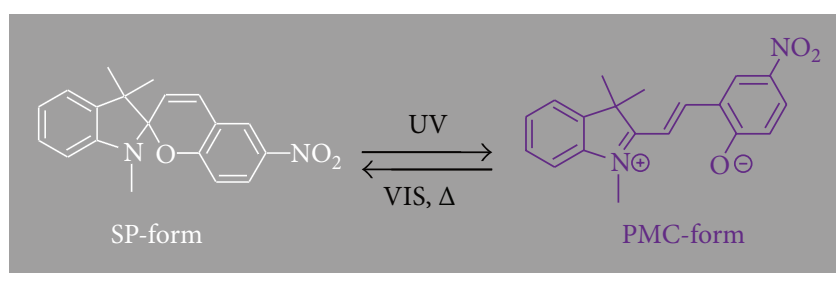

FIGURE 1: Reversible photoisomerization of spiropyran dye $1^{\prime}, 3^{\prime}, 3^{\prime}$ trimethyl-6-nitrospiro[1(2H)-benzopyran-2,2' -indoline] (SP).

photomerocyanine-form (PMC-form) with a high molar absorption coefficient, as shown in Figure 1. The PMCform reverts to the closed-ring isomer (spiropyran-form (SPform)) through a thermally induced ring closure reaction; fading of the colored isomers occurs on a timescale of seconds to minutes at room temperature.

The study reported herein is undertaken to visualize reversibly synchrotron radiation of the superphoton ring-8 $\mathrm{GeV}$ (SPring-8) by using a composite film consisting of a photochromic spiropyran dye that has conversion characteristics of UV-to-visible color and PSP BaFCl:Eu ${ }^{2+}$ particles that have conversion characteristics of X-rays-to-UV emission.

This present study aims to provide a visual recognition support film capable of allowing SPring-8 operators to recognize visually an irradiated position easily and properly with a simple structure that consists of a spiropyran dye and $\mathrm{BaFCl}: \mathrm{Eu}^{2+}$.

\section{Experimental}

A spiropyran dye, $1^{\prime}, 3^{\prime}, 3^{\prime}$-trimethyl-6-nitrospiro[1(2H)-benzopyran-2, $2^{\prime}$-indoline] (SP), was used as a UV light-sensitive photoacceptor, and $\mathrm{BaFCl}: \mathrm{Eu}^{2+}$ was used as a UV-radiation source. SP and BaFCl:Eu ${ }^{2+}$ were purchased from Tokyo Kasei Co. and Nemoto Lumi-Materials Co., respectively. The SP $(45 \mathrm{mg})$ and $\mathrm{BaFCl}: \mathrm{Eu}^{2+}(1500 \mathrm{mg})$ were mixed, and the mixed powder was dissolved at a rate of $30 \mathrm{wt} \%$ to a solution of cyclohexanone and polycarbonate (PC) $(0.16 \mathrm{w} / \mathrm{w}$, $50 \mathrm{~mL}$ ). The suspension was stirred for $10 \mathrm{~min}$. The resulting suspension was applied to a polyethylene terephthalate (PET) substrate using an applicator to produce the SP/BaFCl:Eu ${ }^{2+}$ PC composite film. An aluminum deposition film was prepared on the opposite side of the substrate, and its thickness was $60 \mathrm{~nm}$. The deposition rate was $2 \mathrm{~nm} \mathrm{~s}^{-1}$, and the substrate temperature was maintained at an ambient temperature. The practical photograph of the composite/PET/Al three-layered film, its schematic diagram, and an illustration internal of the structure are shown in Figure 2.

The absorption spectrum of the SP toluene solution was measured with a UV-Vis-NIR spectrophotometer (PerkinElmer Co., Lambda 1050UV/Vis/NIR), and the spectral reflectance of the SP/BaFCl: $\mathrm{Eu}^{2+}$ PC composite film was measured with a spectral colorimeter (Konica Minolta Co., CM-2600d). The powder morphology and particle size of $\mathrm{BaFCl}: \mathrm{Eu}^{2+}$ were characterized using scanning electron microscopy (SEM) (Hitachi S-3000). The BrunauerEmmett-Teller (BET) specific surface area of $\mathrm{BaFCl}: \mathrm{Eu}^{2+}$
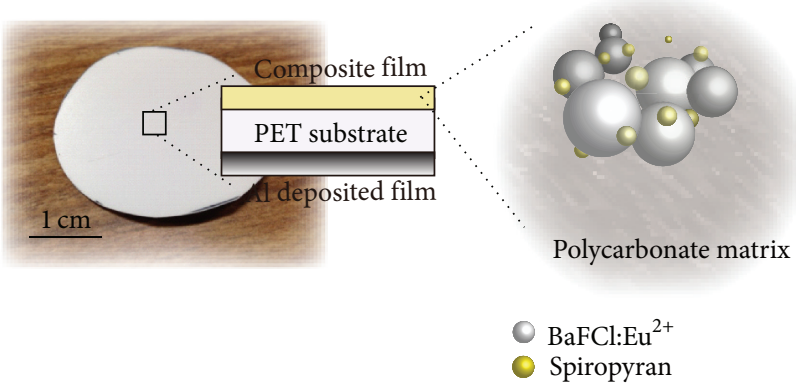

Figure 2: Photograph showing the composite (SP/BaFCl:Eu ${ }^{2+} \mathrm{PC}$ as binder)/PET/Al three-layered film and schematic diagrams of its cross-section and internal structure.

was determined from $\mathrm{Kr}$ adsorption at $77^{\circ} \mathrm{C}$ using a TriStar II 3020 (Shimadzu Co.). The samples were degassed at $500^{\circ} \mathrm{C}$ for $60 \mathrm{~min}$ prior to $\mathrm{Kr}$ adsorption measurement. An X-ray diffractometer (Mac Science Co., MX Labo) with $\mathrm{Cu} \mathrm{K} \alpha$ monochromated X-rays operating at $40 \mathrm{kV}$ and $20 \mathrm{~mA}$ was used to produce X-radiation of $0.154 \mathrm{~nm}$. An $\mathrm{X}$-ray excited optical luminescence (XEOL) spectrum of $\mathrm{BaFCl}: \mathrm{Eu}^{2+}$, which was generated with $\mathrm{X}$-radiation, was guided by a photonic multichannel analyzer (Ocean Optics Co., USB4000) through fused silica fiber (Ocean Optics Co., P400-2-UV-VIS). Synchrotron radiation visualizing was performed at the BL45XU with an undulator at SPring-8. The beam energy and flux were $12.4 \mathrm{keV}$ and $\sim 10^{11}$ photon $\mathrm{s}^{-1}$, and the X-radiation from the SPring- 8 was utilized through a chopper at intervals of $100 \mathrm{~ms}$ (i.e., $100 \mathrm{~ms}$ ON, $100 \mathrm{~ms}$ OFF). The beam size of the incident X-rays on the composite film surface was $200 \mu \mathrm{m} \times 300 \mu \mathrm{m}$. All measurements were carried out at room temperature.

\section{Results and Discussion}

The photochromic reaction of the SP was carried out upon irradiation with $365-375 \mathrm{~nm}$ light of UV-LED $\left(90 \mathrm{~mW} / \mathrm{cm}^{2}\right)$. Before UV light irradiation, the main absorption band centered at $336 \mathrm{~nm}$ (extinction coefficient $\varepsilon=9339 \mathrm{M}^{-1} \mathrm{~cm}^{-1}$ ) of the SP-form appeared in the near UV range of $250-420 \mathrm{~nm}$. This absorption band is assigned to $\pi-\pi^{*}$ transitions of chromene and indoline rings. Upon irradiation with UV light, the toluene solution containing the SP changed from colorless (SP-form) to purple (PMC-form), and a broad absorption band appeared with a maximum at $580 \mathrm{~nm}$. After stopping the UV light irradiation, the absorption band of the PMC-form rapidly decreased for approximately a few seconds at ambient temperature; however, in the case of the SP in the PC composite film, the decay time of the colored PMC-form was prolonged for approximately $5 \mathrm{~min}$ at ambient temperature. This result indicates that the decay time of the colored PMC-form in spiropyrans can be controlled through matrix selection. In fact, we have reported that matrix polarity, steric hindrance, or acidity can control the thermal stability of the colored PMC-form, and the thermal stability is drastically enhanced by the interaction between an ionic zwitterionic structure of PMC-form and a matrix with 


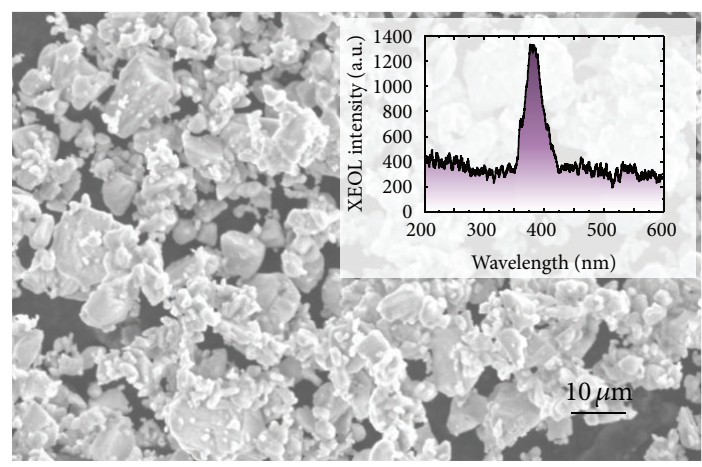

FIGURE 3: SEM image of the BaFCl:Eu ${ }^{2+}$ powder and schematic diagrams of the cross-section and internal structure. The inset shows the XEOL spectrum of $\mathrm{BaFCl}: \mathrm{Eu}^{2+}$ powder under the irradiation of a $0.154 \mathrm{~nm}$ X-ray $(40 \mathrm{kV}, 20 \mathrm{~mA})$.

high polarity [9]. Another important property to this study, the PMC-form, is not produced directly with X-radiation; therefore, the photoisomerization of the SP-to-PMC-form can occur only by irradiation with UV.

The morphology of $\mathrm{BaFCl}: \mathrm{Eu}^{2+}$ crystals was characterized by SEM. From the SEM image (Figure 3), it can be seen that the powder was homogeneous polycrystalline particles with diameters ranging from $5 \mu \mathrm{m}$ to $30 \mu \mathrm{m}$ and an average particle diameter of $13 \mu \mathrm{m}$. To analyze further the BaFCl:Eu ${ }^{2+}$, the BET specific surface area was measured. The BET specific surface area of the BaFCl:Eu ${ }^{2+}$ shows $0.4 \mathrm{~m}^{2} \mathrm{~g}^{-1}$, which indicates that the $\mathrm{BaFCl}: \mathrm{Eu}^{2+}$ particles with a spherical shape that are not porous compare well with the calculated value of $0.1 \mathrm{~m}^{2} \mathrm{~g}^{-1}$ assuming spherical particles. The BaFCl:Eu ${ }^{2+}$ powder can emit near-ultraviolet light when exposed to X-rays, and it has been well known as a photostimulable phosphor (PSP) [10]. The inset in Figure 3 shows that the $\mathrm{BaFCl}: \mathrm{Eu}^{2+}$ powder shows a strong photoluminescence centered at $381 \mathrm{~nm}$ when excited with Xradiation of $0.154 \mathrm{~nm}$ at room temperature. A very small and narrow photoluminescence at $360 \mathrm{~nm}$ is also observed. The $\mathrm{Eu}^{2+}$ ion shows a photoluminescence that strongly depends on its valence state and the nature of the host lattice. The $381 \mathrm{~nm}$ peak is due to the electronic transition from the $4 \mathrm{f}^{6} 5 \mathrm{~d}^{1}\left({ }^{2} \mathrm{e}_{\mathrm{g}}\right)$ state of $\mathrm{Eu}^{2+}$ to the $4 \mathrm{f}^{7}\left({ }^{8} \mathrm{~S}_{7 / 2}\right)$ ground state, and the narrow peak of $360 \mathrm{~nm}$ is attributed to the intraband transition from $4 \mathrm{f}^{7}\left({ }^{6} \mathrm{P}_{7 / 2}\right)$ to $4 \mathrm{f}^{7}\left({ }^{8} \mathrm{~S}_{7 / 2}\right)$ [11]. Accordingly, the strong photoluminescence of $381 \mathrm{~nm}$ overlaps with the region in which SP can be photoisomerized; thus, the $\mathrm{BaFCl}: \mathrm{Eu}^{2+}$ powder is an appropriate combination with the SP for its photoisomerization.

Figure 4 shows the reflectance spectra of the SP/ BaFCl: $\mathrm{Eu}^{2+}$ PC composite film before and after synchrotron radiation at room temperature. Before synchrotron radiation, the color of the composite film is white, derived from the colorless SP-form and the white BaFCl:Eu ${ }^{2+}$. Upon synchrotron radiation for $3 \mathrm{~s}$, the composite film changed from white to deep purple, and a broad reflectance band appeared with a maximum at $580 \mathrm{~nm}$, which is assigned to the $\pi-\pi^{*}$ transitions of the PMC-form. The result implies that

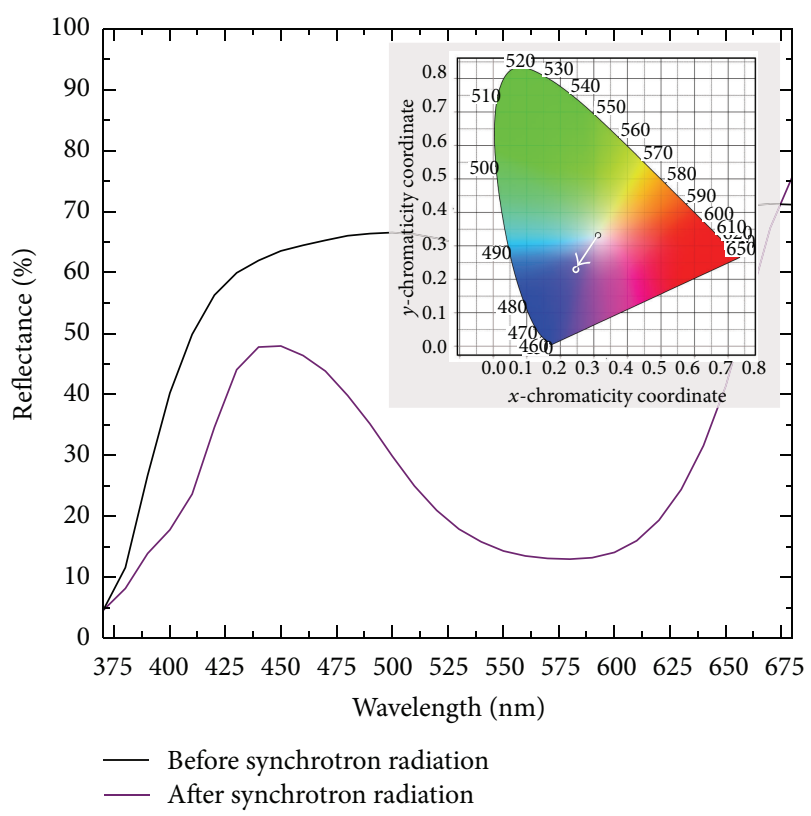

FIGURE 4: Reflectance spectra of the SP/BaFCl:Eu ${ }^{2+}$ PC composite film before and after synchrotron radiation for $2 \mathrm{~s}$. The inset shows color shift in the CIE1931 chromaticity diagram at the maximum brightness. Color coordinates of the composite film are shown as open circles.

the photoisomerization of the SP/BaFCl:Eu ${ }^{2+} \mathrm{PC}$ composite film is accomplished indirectly by absorbing the XEOL derived from BaFCl:Eu ${ }^{2+}$ in the composite film. The SP/BaFCl:Eu ${ }^{2+} \mathrm{PC}$ composite film shows a high visualization property to the synchrotron radiation of SPring-8, which is also suggested from the color difference Commission Internationale de l'Eclairage (CIE) chromaticity diagram of the SP/BaFCl:Eu ${ }^{2+}$ PC composite film before and after synchrotron radiation. For this color change, the CIE chromaticity coordinates $(x, y)$ are found to be from $(0.31,0.33)$ to $(0.24,0.22)$, corresponding to white and deep purple, respectively (the inset in Figure 4). Furthermore, the color change is also expressed using the color space CIE $L^{*} a^{*} b^{*}$, which was calculated from the spectral reflectance that the spectral colorimeter measured. The color changes in terms of the CIE $L^{*} a^{*} b^{*}$ are as follows: $L^{*}$ is 83.76 to $52.12 ; a^{*}$ is -1.43 to 12.39 ; and $b^{*}$ is 0.92 to 33.51 ; thus, the magnitude of total color difference $\Delta E^{*} a b$ is calculated to be +48.76 . $\Delta E^{*} a b$ is defined by the equation $\Delta E^{*} a b=\left[\left(\Delta L^{*}\right)^{2}+\left(\Delta a^{*}\right)^{2}+\right.$ $\left.\left(\Delta b^{*}\right)^{2}\right]^{1 / 2}$, where $\Delta L^{*}$ is the lightness difference, $\Delta a^{*}$ is the red/green difference, and $\Delta b^{*}$ is the yellow/blue difference. Consequently, the total color difference $\Delta E^{*} a b$ of the SP/ $\mathrm{BaFCl}: \mathrm{Eu}^{2+} \mathrm{PC}$ composite film can be easily recognized. More-over, the colored area disappeared for approximately a few hours, so the color change can be used many times repeatedly.

A practical application of visualization for synchrotron radiation using the composite film is shown in Figure 4. Each picture in Figure 5 shows the visualization appearance for synchrotron radiation at an interval of $100 \mathrm{~ms}$ 


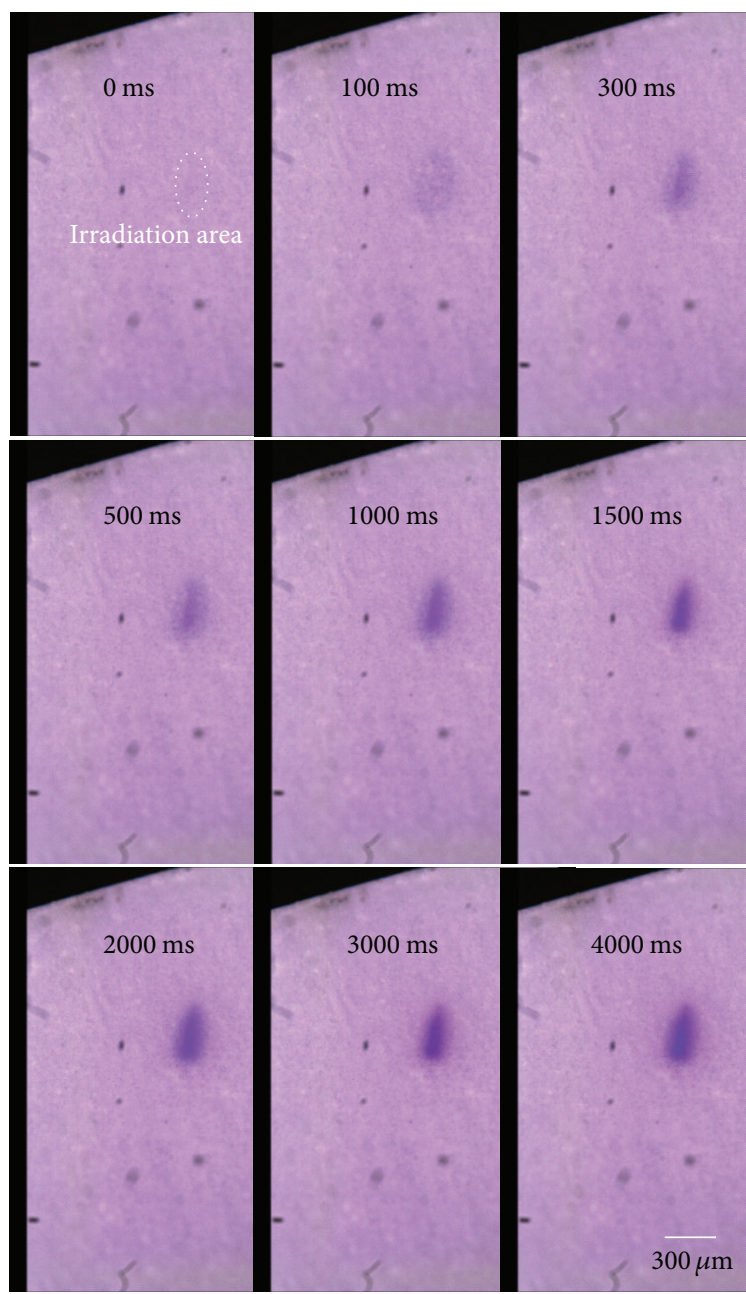

FIGURE 5: Photographs showing visualization for the synchrotron radiation of SPring- 8 using the SP/BaFCl:Eu ${ }^{2+} \mathrm{PC}$ composite film. Deep purple areas indicate the spot that is irradiated with the synchrotron radiation of SPring-8.

(see Movie S1 of the Supplementary Material available online at http://dx.doi.org/10.1155/2014/236382). The deep purple area indicates the synchrotron radiation area, which is in good agreement with the beam size of the synchrotron radiation, and the pale pink area (the actual color is white, and the visual change is due to room lighting) is the unirradiated area. The color intensity of the deep purple area visualized by synchrotron radiation is saturated within $3000 \mathrm{~ms}$; thus, the photostationary state is reached within $2000 \mathrm{~ms}$. In addition, bleaching of the composite film was confirmed for a few minutes in an ambient temperature; the visible absorption band of the PMC-form had completely disappeared. The visualization for synchrotron radiation was repeated a few dozen times with depression of the absorption intensity of the PMC-form to less than $30 \%$. The visualization synchrotron radiation using the composite film shows very similar characteristics to the GAFCHROMIC EBT2 dosimetry film which has been designed specifically as a time-and-cost-saving tool that addresses the needs of medical physicists and dosimetrists working in radiotherapy environments; however, the reusable visualizing characteristics of the $\mathrm{SP} / \mathrm{BaFCl}: \mathrm{Eu}^{2+} \mathrm{PC}$ composite film is significantly different from the commercially available dosimetry films. Furthermore, the visualization property of the SP/BaFCl: $\mathrm{Eu}^{2+} \mathrm{PC}$ composite film is one possible way to the detection of radiation rays of radioactive materials and will provide efficient color change in accordance with the intensity of the absorbed dose.

\section{Conclusions}

We successfully demonstrated the reversible visualization of synchrotron radiation by using the composite film comprised of a photochromic SP with the conversion characteristics of UV-to-visible color and PSP BaFCl:Eu ${ }^{2+}$ particles with the conversion characteristics of X-rays-to-UV emission.

\section{Conflict of Interests}

The authors declare that there is no conflict of interests regarding the publication of this paper.

\section{Acknowledgments}

This work was financially supported by JSPS Grant-in-Aid for Young Scientists (B) 26820305 and NISSHA Printing Co., Ltd. The synchrotron radiation experiments were performed at BL45XU in SPring-8. The author Kenji Kinashi acknowledges Takashi Nishino and Chizuru Hongo at Kobe University for measuring the BET specific surface area.

\section{References}

[1] G. Blasse and B. C. Grabmaier, "X-ray phosphors and scintillators (integrating techniques)," in Luminescent Materials, chapter 8, pp. 146-169, Springer, Berlin, Germany, 1994.

[2] M. Sonoda, M. Takano, J. Miyahara, and H. Kato, "Computed radiography utilizing scanning laser stimulated luminescence," Radiology, vol. 148, no. 3, pp. 833-838, 1983.

[3] S. Schweizer, "Physics and current understanding of X-ray storage phosphors," Physica Status Solidi (a), vol. 187, no. 2, pp. 335-393, 2001.

[4] J. C. Crano, W. S. Kwak, and C. N. Welch, "Spirooxazines and their use in photochromic lenses," in Applied Photochromic Polymer Systems, C. B. McArdle, Ed., chapter 2, p. 31, Blackie, London, UK, 1992.

[5] G. Berkovic, V. Krongauz, and V. Weiss, "Spiropyrans and spirooxazines for memories and switches," Chemical Reviews, vol. 100, no. 5, pp. 1741-1753, 2000.

[6] I. Shimizu, H. Kokado, and E. Inoue, "Photoreversible photographic systems. V. Reverse photochromism of (photospiran/acid) system in acetone," Bulletin of the Chemical Society of Japan, vol. 42, no. 6, pp. 1726-1729, 1969.

[7] I. Shimizu, H. Kokado, and E. Inoue, "Photoreversible photographic systems. VI. Reverse photochromism of 1,3,3trimethylspiro[indoline-2,2l-benzopyran]-8I-carboxylic acid," Bulletin of the Chemical Society of Japan, vol. 42, no. 6, pp. 1730$1734,1969$. 
[8] F. M. Raymo and S. Giordani, "Signal processing at the molecular level," Journal of the American Chemical Society, vol. 123, no. 19, pp. 4651-4652, 2001.

[9] K. Kinashi, S. Nakamura, Y. Ono, K. Ishida, and Y. Ueda, "Reverse photochromism of spiropyran in silica," Journal of Photochemistry and Photobiology A: Chemistry, vol. 213, no. 2-3, pp. 136-140, 2010.

[10] Q. Ju, Y. Liu, R. Li, L. Liu, W. Luo, and X. Chen, "Optical spectroscopy of $\mathrm{Eu}^{3+}$-doped $\mathrm{BaFCl}$ nanocrystals," The Journal of Physical Chemistry C, vol. 113, no. 6, pp. 2309-2315, 2009.

[11] W. Chen, N. Kristianpoller, A. Shmilevich, D. Weiss, R. Chen, and M. Su, "X-ray storage luminescence of $\mathrm{BaFCl}: \mathrm{Eu}^{2+}$ single crystals," Journal of Physical Chemistry B, vol. 109, no. 23, pp. 11505-11511, 2005. 

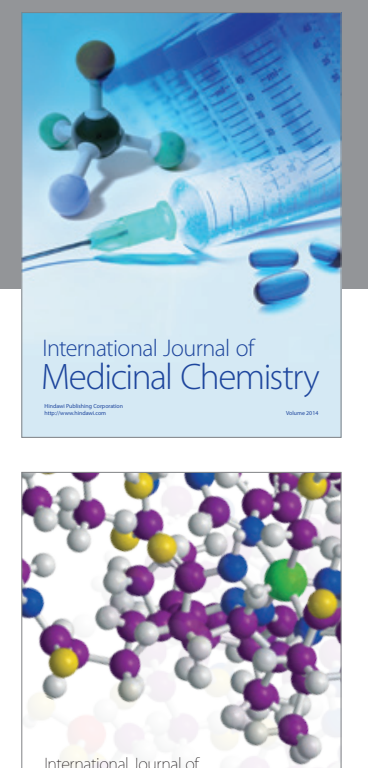

\section{Carbohydrate} Chemistry

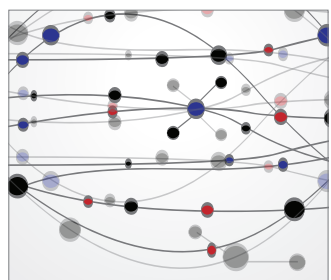

The Scientific World Journal
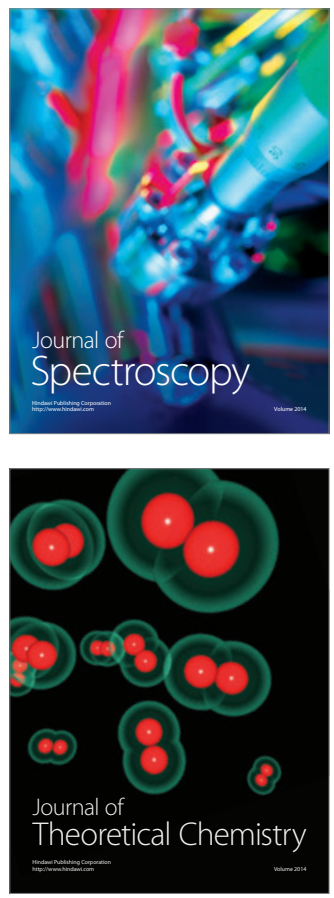
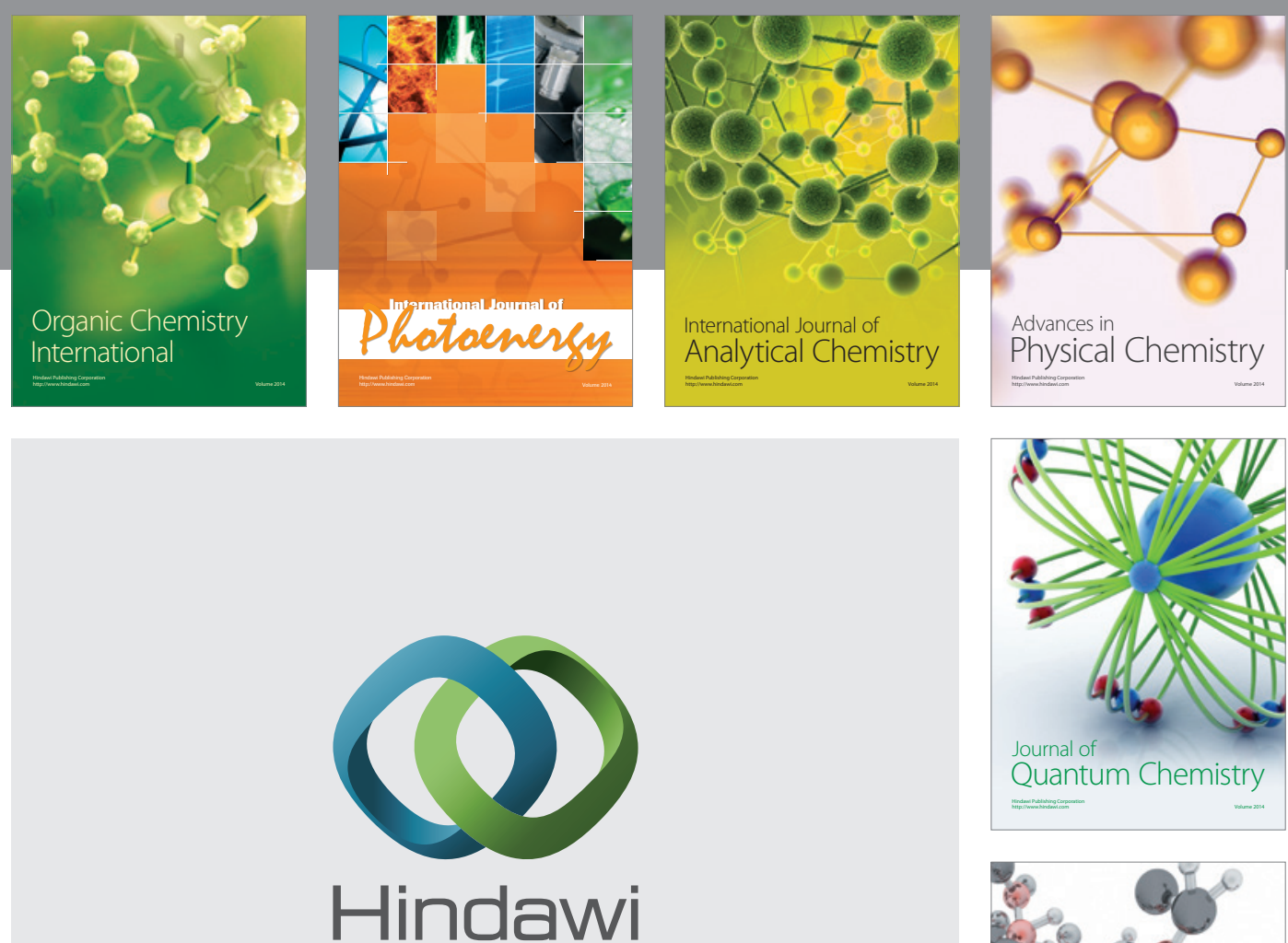

Submit your manuscripts at

http://www.hindawi.com

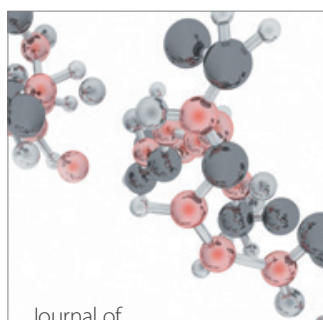

Analytical Methods

in Chemistry

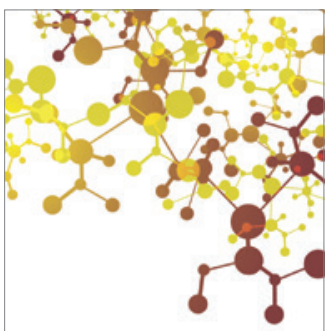

Journal of

Applied Chemistry

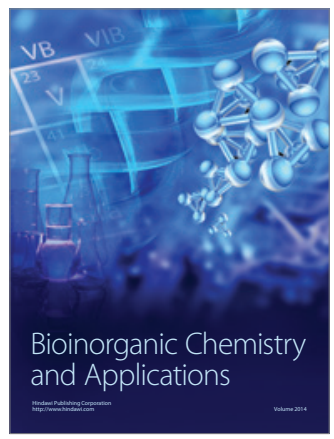

Inorganic Chemistry
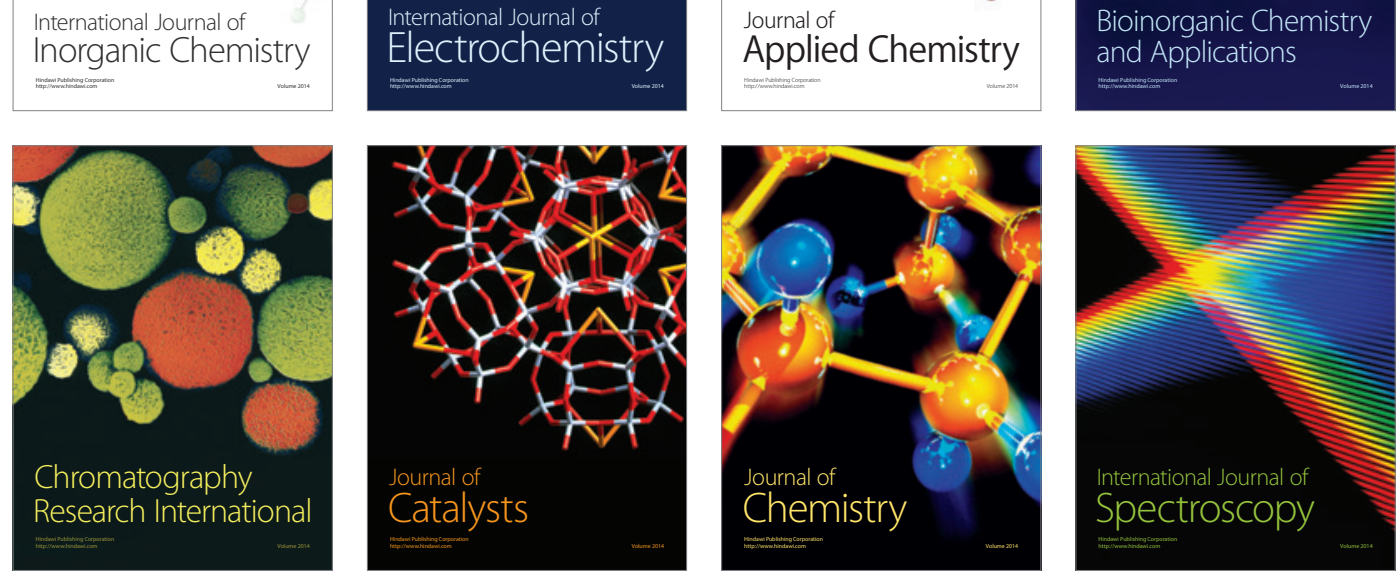